\title{
Metagenomic Analysis Revealing Antibiotic Resistance Genes (ARGs) and Their Genetic Compartments in the Tibetan Environment
}

Baowei Chen, ${ }_{\dagger}^{\dagger,+\#}$ Ke Yuan, ${ }^{\perp}$ Xin Chen, ${ }^{\dagger, \#}$ Ying Yang, ${ }^{\dagger}$ Tong Zhang, ${ }^{\S}$ Yawei Wang,,$"$ Tiangang Luan, ${ }^{\perp}$ Shichun Zou, ${ }^{\dagger}$ and Xiangdong $\mathrm{Li}^{*}$,

†South China Sea Resource Exploitation and Protection Collaborative Innovation Center, School of Marine Sciences, Sun Yat-sen University, Guangzhou 510275, China

${ }^{\star}$ Department of Civil and Environmental Engineering, The Hong Kong Polytechnic University, Hung Hom, Kowloon Hong Kong ${ }^{\S}$ Environmental Biotechnology Laboratory, The University of Hong Kong, Pokfulam, Hong Kong

"State Key Laboratory of Environmental Chemistry and Ecotoxicology, Research Center of Eco-Environmental Sciences, Chinese Academy of Sciences, Beijing 100085, China

${ }^{\perp}$ MOE Key Laboratory of Aquatic Product Safety, School of Life Sciences, Sun Yat-Sen University, Guangzhou 510275, China

${ }^{\#}$ Guangdong Provincial Key Laboratory of Marine Resources and Coastal Engineering, School of Marine Sciences, Sun Yat-Sen University, Guangzhou 510275, China

Supporting Information

ABSTRACT: Comprehensive profiles of antibiotic resistance genes (ARGs) and mobile genetic elements (MGEs) in a minimally impacted environment are essential to understanding the evolution and dissemination of modern antibiotic resistance. Chemical analyses of the samples collected from Tibet demonstrated that the region under investigation was almost devoid of anthropogenic antibiotics. The soils, animal wastes, and sediments were different from each other in terms of bacterial community structures, and in the typical profiles of ARGs and MGEs. Diverse ARGs that encoded resistance to common antibiotics (e.g., beta-lactams, fluoroquinolones, etc.) were found mainly via an efflux mechanism completely distinct from modern antibiotic resistome. In addition, a very small fraction of ARGs in the Tibetan environment were carried by MGEs, indicating the low potential of these ARGs to be transferred among bacteria. In comparison to the ARG profiles in relatively pristine Tibet, contemporary ARGs and MGEs in human-impacted environments have evolved substantially since the broad use of anthropogenic antibiotics.

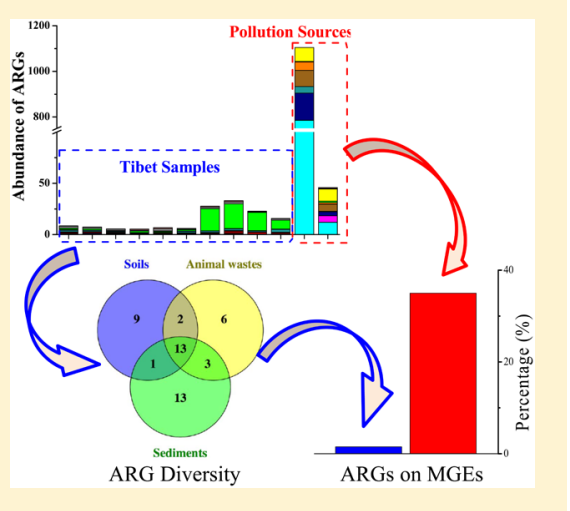

\section{INTRODUCTION}

In the realm of public health in the last century, antibiotics are a story of success. Once-high mortality rates from bacterial infectious diseases plunged. ${ }^{1}$ Trust in the efficacy of antibiotics led to their widespread use in human therapies, husbandry, and aquaculture. However, antibiotic resistance is now prevalent in modern environmental and human commensal microbes, ${ }^{2-4}$ while infectious diseases are still the second-leading cause of death worldwide. ${ }^{5}$ The scientific community has begun to ponder why antibiotic resistance is so common and widespread in modern environments that some antibiotics are no longer effective in some clinical cases, and treatment options for certain pathogens have become very limited. ${ }^{6}$ Collections of microflora that predate the antibiotic age are highly sensitive to antibiotics, while their mobile genetic elements (MGEs) rarely harbor resistance genes. ${ }^{7,8}$ In modern environments, antibiotics for anthropogenic use have served as the major selective pressure in the proliferation of antibiotic-resistant bacteria, as well as a driving force for bacteria to evolve and acquire antibiotic resistance. ${ }^{9}$
Antibiotic resistance inherently exists in natural environments. Indeed, a large variety of ARGs are carried by microbes that inhabit various niches where there is no anthropogenic impact, e.g., isolated caves, ${ }^{10}$ deep oceans, ${ }^{11}$ and deep terrestrial subsurface. ${ }^{12}$ In fact, microbes can secrete antibiotics as a competitive mode between microbes, ${ }^{13}$ and the microbial synthetic pathways of antibiotics have evolved over millions of years. ${ }^{14}$ Recently, a marine-derived actinomycete was shown to produce a novel natural antibiotic. ${ }^{15}$ Soil bacteria have exhibited the ability to grow on antibiotics as the sole source of carbon. ${ }^{16}$ Whether microbes are antibiotic producers or users, they must develop effective resistance mechanisms to protect themselves from antibiotics. ${ }^{17,18}$ The genes associated with different

Special Issue: Jerry Schnoor's Lasting Influence on Global and Regional Environmental Research

Received: February 4, 2016

Revised: April 19, 2016

Accepted: April 25, 2016

Published: April 25, 2016 
antibiotic resistance mechanisms have evolved and been maintained in microbes over a long period of time. ${ }^{19}$ Therefore, antibiotic resistance is a natural and ancient phenomenon.

In combination with a massive release of ARGs and ARB from significant sources of pollution into nearby environments, ${ }^{20,21}$ MGEs (e.g., integrons, plasmids, etc.) facilitate horizontal gene shuttles of ARGs between gene locations or between bacterial hosts under external stress. ${ }^{22,23}$ As a result, it could be especially difficult to retrieve the baseline profiles of ARGs and MGEs prior to the antibiotic era from such a complicated situation. Nevertheless, an alternative approach can be to study ARGs in different habitats of the pristine environment, which would provide good insights into the provenance and evolution of modern resistome. The Tibetan environment is unique, as indigenous bacteria have never or have hardly been exposed to anthropogenic antibiotics and ARGs. The occurrence and prevalence of antibiotic resistance in this region is not yet well documented. Exploring these baseline resistance profiles of nearly intact environments can indicate from what initial state the evolution and development of contemporary antibiotic resistome began. Furthermore, although the role of MGEs in the spread of ARG has been established in human-impacted settings, the situation might be different in a pristine environment. ${ }^{24}$ Characterizing MGEs and genetic compartments of ARGs in different environmental habitats of Tibet could be helpful in elucidating the potential transferability of ARGs in the minimally impacted environment.

The aim of the present study is to compile comprehensive profiles of ARGs and their genetic locations in the soils, animal wastes, and sediments collected from the pristine Tibetan environment using a state-of-art metagenomic method, and to elucidate the baseline and primitive state of antibiotic resistance with minimal exposure of anthropogenic antibiotics.

\section{MATERIALS AND METHODS}

Study Area and Sampling. Lake Namco (N30 $30^{\prime}-30^{\circ} 35^{\prime}$, $\mathrm{E} 90^{\circ} 16^{\prime}-91^{\circ} 03^{\prime}, 4718 \mathrm{~m}$ a.s.l.) is situated at the center of the Tibetan Plateau. The lake has a surface area of about $1982 \mathrm{~km}^{2}$ and a depth exceeding $90 \mathrm{~m} \cdot{ }^{25,26}$ It is mainly supplied from precipitation and glacier meltwater from the Nyainqen Tanglha Range in the southeast. The water loss is entirely via natural evaporation because the lake is enclosed. The mean values of the temperature, $\mathrm{pH}$, dissolved oxygen, and electric conductivity of its surface water are approximately $11.43{ }^{\circ} \mathrm{C}, 9.21$, $8.90 \mathrm{mg} \mathrm{L}^{-1}$, and $1851 \mu \mathrm{S} \mathrm{cm} \mathrm{cm}^{-1}$, respectively. Only a few thousands of people live on the pastures around Lake Namco, and there is little industry in this region. Qiangyong Glacier Lake (N28 $53.390^{\prime \prime}, \mathrm{E} 90^{\circ} 496^{\prime}, 4855 \mathrm{~m}$ a.s.l.) is located in the southern part of the Tibetan Plateau, in the Indian monsoon climate region, and is comprised of a small lake (area, $0.03 \mathrm{~km}^{2}$; maximum water depth, $3.5 \mathrm{~m}$ ) and a large lake (area, $0.1 \mathrm{~km}^{2}$; maximum water depth, $30 \mathrm{~m}){ }^{27}$ It is less than $1 \mathrm{~km}$ from the tongue of the glacier. Most of its water comes from the meltwater from the upper part of the Qiangyong glacier. The temperature, $\mathrm{pH}$, and electric conductivity of surface water are on average $6.89^{\circ} \mathrm{C}, 8.32$, and $136.4 \mu \mathrm{S} \mathrm{cm}^{-1}$, respectively. No people inhabit the area near Qiangyong Glacier Lake. Therefore, the regions around both Namco Lake and Qiangyong Glacier Lake are subjected to very little or no anthropogenic impacts.

Seven samples were collected from the Chinese Academy of Sciences' Namco Monitoring and Research Station for Multisphere Interactions (N30 $46.44^{\prime}$, E90 $59.31^{\prime}, 4730 \mathrm{~m}$ a.s.l.) in August of 2013, including one sediment, two animal feces (yak and sheep), and two soil samples. As a comparison, two sediment samples were collected from Qiangyong Glacier Lake (N28 ${ }^{\circ} 53.489^{\prime}$, E90 ${ }^{\circ} 13.582^{\prime}$, $4796 \mathrm{~m}$ a.s.l.). Sediment samples were taken using a grab sampler, and the top $5 \mathrm{~cm}$ of soil samples were taken using a sampling shovel. Following the collection, all samples were stored in sterilized polyethylene plastic bags. All samples were immediately kept in a refrigerator at $-20{ }^{\circ} \mathrm{C}$ until DNA was extracted and an analysis for common antibiotics was carried out in the laboratory.

Sewage treatment plants and husbandry are considered typical sources of ARGs pollution. ${ }^{28,29}$ In the present study, an active sludge sample was collected from a sewage treatment plant in Shatin, Hong Kong, in July $2007 ;^{28}$ and a swine feces sample was taken from a 7-8 month-old pig in a swine feedlot in Zhuhai, China in January 2013. ${ }^{30}$ The two samples that represented as typical pollution sources of ARGs due to the use of anthropogenic antibiotics were also compared with those collected from Tibet.

Analysis of Antibiotics. Ten commonly used antibiotics, including norfloxacin and ofloxacin (fluoroquinolones), sulfacetamide, sulfadiazine, sulfamethazine, and sulfamethoxazole (sulfonamides), tetracycline (tetracyclines), and clarithromycin, erythromycin and roxithromycin (macrolides) were analyzed in this study. Detailed methods of pretreating and analyzing antibiotics have been described in our previous publications. ${ }^{21,31}$ In brief, the extraction and purification of antibiotics in the samples were performed through tandem solid-phase extraction (SPE) on SAX (6 mL, $200 \mathrm{mg}, \mathrm{CNW}$, Germany) and HLB cartridges $(6 \mathrm{~mL}, 500 \mathrm{mg}$, Waters, U.K.). The final extract was analyzed using an Agilent HP1100 liquid chromatography (Agilent, Palo Alto, CA) along with Applied Biosystems API 4000 tandem mass spectrometry (HPLC MS/ MS). The chromatographic separation of antibiotics was conducted using an Agilent ZORBAX C18 column (2.1 $\AA$ $150 \mathrm{~mm}, 5 \mu \mathrm{m}$ particle size). Following the separation, the elution was introduced into a mass spectrometer for the determination of antibiotics.

Potential losses during the analytical procedure were monitored using ${ }^{13} \mathrm{C}_{3}$-caffeine as a surrogate standard. Recoveries of ${ }^{13} \mathrm{C}_{3}$-caffeine from all samples were higher than $75 \%$. Recoveries of target antibiotics from samples were determined at two spiking concentrations $(5$ and $10 \mathrm{ng} / \mathrm{g}$ for samples) with four replicates. The recoveries of macrolides, sulfonamides and tetracyclines were greater than $80 \%$, and the recoveries of fluoroquinolones were approximately 50\%. The relative standard deviations (RSDs) for all antibiotics were lower than $10 \%$. The limits of quantification (LOQ) were determined as the minimum detectable concentration of an analyte with a signal-to-noise ratio $(\mathrm{S} / \mathrm{N})$ of 10 . The LOQs of the target antibiotics in the samples were $0.04-4.50 \mathrm{ng} / \mathrm{g}$.

DNA Extraction and High-Throughput Sequencing. DNA was extracted from the samples using the PowerSoil DNA Isolation Kit (MOBIO, CA) according to the manufacturer's instructions. DNA preparation was performed multiple times due to the low content of DNA in some samples, and because combining DNA extractant can minimize possible heterogeneity in the samples and avoid the potential bias arising from the process of extracting a single DNA sample. The purity and concentration of the DNA were measured using a Thermo Scientific NanoDrop1000 Spectrophotometer. DNA from each of the samples was delivered to the Beijing Genomics Institute (BGI) (Shenzhen, China) for sequencing. Approximately $5 \mu \mathrm{g}$ 
of DNA was sheared into $\sim 180$ bp fragments. Then the overhangs resulting from fragmentation were end-repaired using T4 DNA polymerase, Klenow Fragment and T4 Polynucleotide Kinase. After adding an 'A' base to the 3 ' end of the blunt DNA fragments, adapters were ligated to the ends of the DNA fragments. The desired fragments were purified using gel-electrophoresis, and selectively enriched and amplified by PCR. The index tags were introduced into the adapter to construct DNA libraries at the PCR stage. The qualified DNA libraries were used for sequencing by Illumina HiSeq 2000. The total data output of the 10 samples exceeded $30 \mathrm{~Gb}$. The size of the data for each sample was approximately $3.2 \mathrm{~Gb}$. Raw sequencing data was deposited on the GenBank and MGRAST, and accession number was presented in Table S1 (Supporting Information (SI)).

Bioinformatic Analysis. The raw reads (100 bp in length) of each data set were first trimmed to discard low-quality reads that contained ambiguous nucleotides or a quality value of lower than 20 using a customized python script. Trimmed clean reads were overlapped into longer sequence tags according to the following parameters: a length of at least $20 \mathrm{nt}$ of overlapping region was required, and at most two mismatches were allowed. The SILVA Small Subunit (SSU) database (version 10.4) was used to characterize the structure of the bacterial community through a local BLASTN program with an $E$-value cutoff of $10^{-20} .^{32,33}$ The $16 \mathrm{~S}$ rRNA gene-like sequences from the BLAST results were then assigned to NCBI taxonomies with MEGAN (version 4.70.4) using the Lowest Common Ancestor (LCA) algorithm, where the absolute cutoff was a BLAST bitscore of 50 and the relative cutoff was $10 \%$ of the top 50 hits. ${ }^{34}$ The BLAST program (Linux Release 2.2.29) was downloaded from the NCBI, and run on the local sever to search target sequences from our sequencing data sets. The local BLASTX program was used to align the trimmed clean tags against an antibiotic resistance genes database (ARDB). ${ }^{35}$ A tag was determined to have an ARG-like sequence if the best BLASTX hit had a similarity of higher than $90 \%$ and an alignment length of more than 25 amino acids (aa). ${ }^{28}$ The local BLASTN program was employed to align our sequencing data against the databases of integrons and plasmids for characterizing these two kinds of MGEs in the samples. An integron database was constructed according to the nucleotide sequences of all integrases available in the INTEGRALL database (1447 integrase genes and 8053 gene cassettes). ${ }^{36}$ Meanwhile, a plasmid database was developed on the basis of the plasmid sequences of the NCBI RefSeq database (2465 sequences). ${ }^{28} \mathrm{~A}$ tag was annotated as an integron-like sequence if the nucleotide sequence identity of the best BLASTN hit was higher than $90 \%$ with an alignment length of at least $50 \mathrm{bp}$, and the plasmid-like tags in all data sets were determined by the alignments with a nucleotide sequence identity of above $95 \%$ over a length of at least $90 \mathrm{bp}$.

Statistical Analysis. One-way analysis of variance (ANOVA), hierarchical cluster analysis (HCA) and principal component analysis (PCA) were conducted using SPSS for Windows Release 16.0 (SPSS Inc. U.S.). The ANOVA was used to analyze the differences in the abundance and profiles of ARGs and MGEs between samples. Differences at the $p<0.01$ level (99\% confidence interval) were considered statistically significant. HCA was carried out to cluster samples, where paired group algorithm and Euclidean distance were used. The samples were also characterized and grouped according to relative percentages of resistance types using PCA methods, where all sequencing data sets were used as statistical cases $(n=$ $12)$ and antibiotic resistance types were used as the variables $(n$ $=15$ ).

\section{RESULTS AND DISCUSSION}

Bacterial Community Structures in the Soils, Animal Wastes, and Sediments collected from Tibet. The $16 \mathrm{~S}$ rRNA genes were extracted by sequence alignment against the SSU database. The analysis of bacterial community structures was completed using MEGAN. The results demonstrated that bacteria in the soils, animal wastes, and sediments were assigned to 15,15 , and 19 phyla, respectively. Figure $1 \mathrm{~A}$
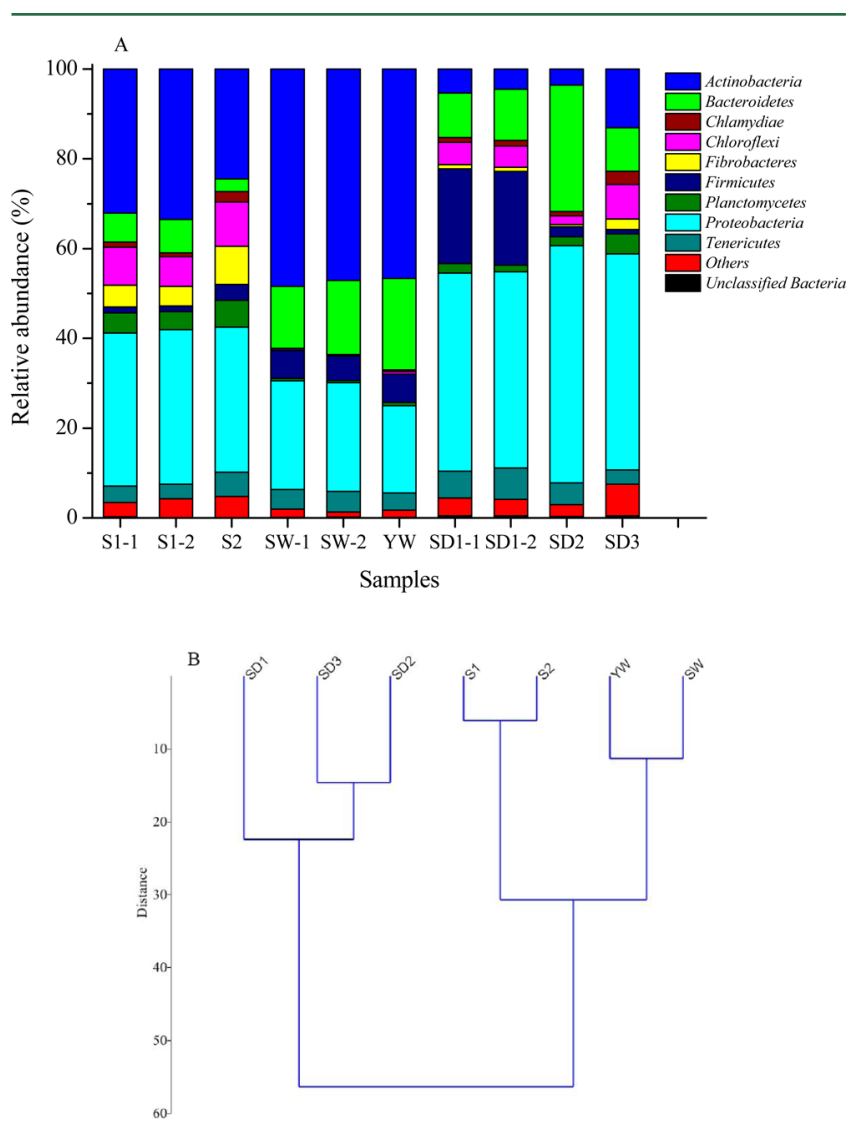

Figure 1. Bacterial community structures at the phylum level in Tibet (A) and a sample classification using hierarchical cluster analysis (B). The S, SW, YW, and SD represent soils, sheep waste, yak waste, and sediments, respectively. The number $(-1$ or -2$)$ at the end of sample identities indicates that a duplicate sequencing analysis was performed for these samples. "Others" indicates the sum of the relative percentages of phyla that were lower than $2 \%$ in each sample.

showed that relative abundance of these phyla varied greatly among the soils, animal wastes, and sediments in Tibet. Actinobacteria was highly abundant in the animal wastes, while its relative abundance was lower in the soils than in the animal wastes. In contrast, Proteobacteria was more enriched in the sediments than in other samples. The samples were further characterized using HCA, where relative percentages of bacterial phyla in each of the samples were employed as data input (Figure 1B). In general, the samples were significantly separated and clustered according to the structure of the bacterial community, which was in good accordance with the sample types. 


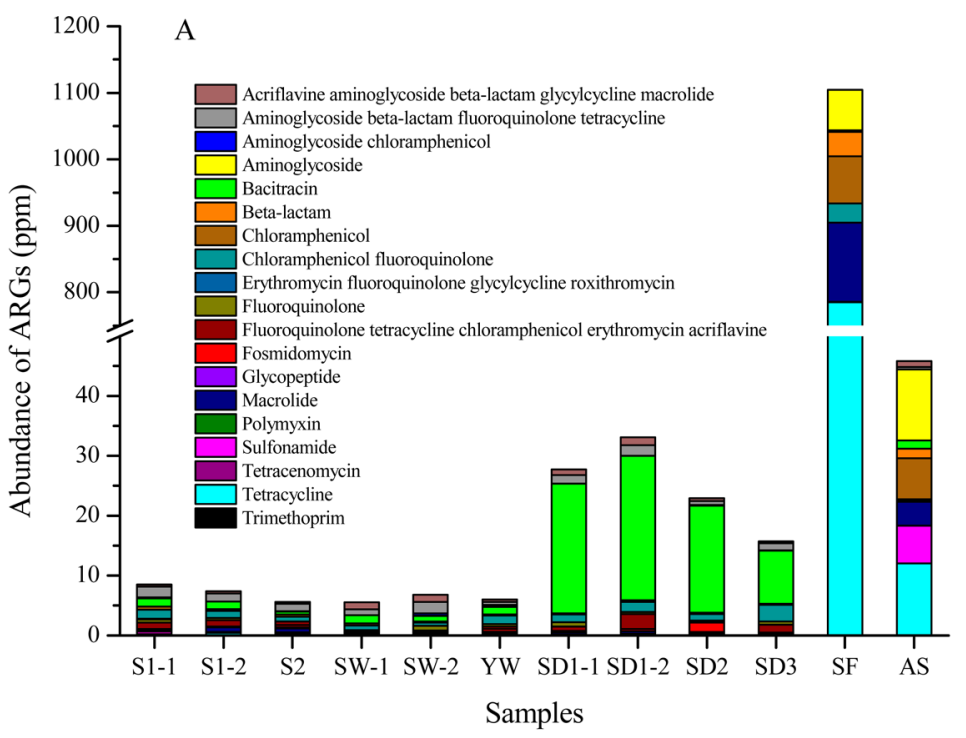

B Beta_lactam resistantance genotypes
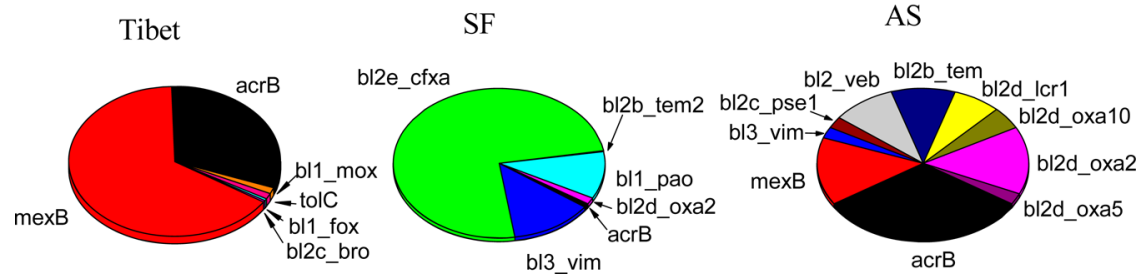

Figure 2. Comparisons of ARG abundance and resistance types (A) and the beta-lactam resistance gene profile (B) among samples of Tibet and representative sources of ARG pollution. Abundance of ARGs was normalized to the size of the sequencing data, and "ppm" refers to one ARGs-like tag in one million metagenomic sequencing tags. SF and AS represent swine feces and active sludge, respectively. Other sample identities are the same as those in Figure 1. In Figure 2B, subtypes represent the types of antibiotic resistance genes described in the ARDB database.

The Tibetan Plateau is considered a unique and important permafrost environment. ${ }^{38}$ However, the knowledge of bacterial communities in various environmental habitats of Tibet is limited. The 454 pyrosequencing-based approaches used in previous studies showed that Actinobacteria and Proteobacteria phyla were dominant in the permafrost soils of the Tibetan Plateau, which was completely consistent with the findings in the present study. ${ }^{39,40}$ Most of the bacterial isolates from the sediments in Tibet were affiliated with Proteobacteria. ${ }^{38}$ Culture-independent approaches also demonstrated that bacterial 16S rRNA gene clones in the sediments of the same lake were mainly assigned to Proteobacteria. ${ }^{25,41,42}$ To our knowledge, bacterial communities in Tibetan animal wastes have not yet been explored. This study demonstrated the predominance of Actinobacteria in animal wastes of Tibet, which was consistent with that ruminant feces often harbored diverse symbiotic Actinobacteria. ${ }^{43}$ The samples collected from Tibet could be characterized and differentiated by typical patterns of composition and the relative abundance of bacterial communities. Lake sediments, soils, and animal wastes were conceptualized as an aquatic habitat, terrestrial habitat, and biological habitat, respectively.

Occurrence of Common Antibiotics in Tibet. Ten common antibiotics belonging to four groups (sulfonamides, tetracyclines, fluoroquinolines, and macrolides) in the soils, animal wastes, and sediments of Tibet were analyzed using HPLC-MS/MS. The target antibiotics were not detectable in any of the samples collected from Tibet. The results suggested that this region under investigation has minimally been exposed to detectable anthropogenic antibiotics, and that the bacterial inhabitants of this region have also not been suffered from the significant impacts of the wide use of antibiotics.

Abundance and Resistance Types of ARGs in Tibet. The ARGs found in the soil, animal waste, and sediment samples of Tibet were sorted according to the types of antibiotics toward which they exhibited resistance (viz. resistance type), as shown in Figure $2 \mathrm{~A}$ and Figure S1 (SI). In order to eliminate the bias caused by sequencing depth, the number of ARG-like tags in each of the samples was normalized to the total number of tags of the same sample. A duplicate analysis of S1, SW, and SD1 demonstrated good repeatability in the metagenomic profiling of ARGs, with a relative standard deviation (RSD) of less than $10 \%$. ARGs were significantly more abundant in the sediments than in the soils or animal wastes $(p<0.01)$, and no significant difference was observed between the soils and animal wastes. The ARGs in the pristine environment of the Tibet were associated with more than 10 groups of antibiotics, including commonly used antibiotics (e.g., aminoglycosides, beta-lactams, fluoroquinolones, macrolides, etc.) and less popular antibiotics (e.g., bacitracin, polymyxin, etc.). A resistance gene specific to bacitracin was detectable in all of the samples, and was highly abundant in the sediments. In contrast, a larger part of ARGs in Tibet conferred simultaneous resistance to multiple antibiotics, and these accounted for at 

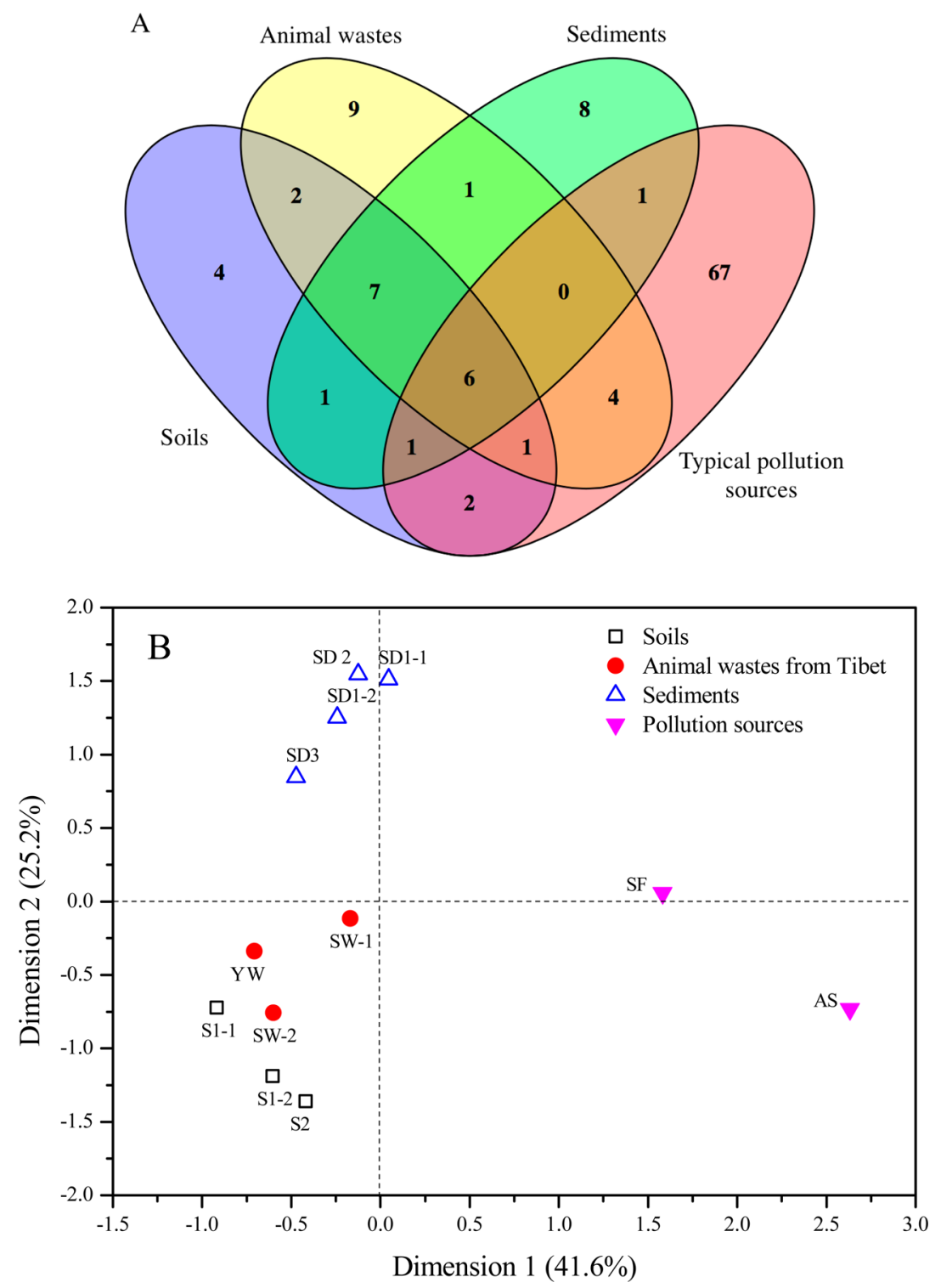

Figure 3. Venn diagram of antibiotic resistance subtypes (A) and PCA analysis of ARG profiles (B) in different environmental habitats of Tibet and representative sources of pollution. Number in each of the color blocks indicates the number of subtypes of ARGs that could be identified in single or multiple types of samples. Relative percentages of resistance types in the samples were used for the PCA analysis. Typical pollution sources represent swine feces and active sludge that were collected from husbandry operations and sewage treatment plants, respectively. The sample identities are the same as those in Figure 1 and 2

least $80 \%$ of the resistance genes related to common antibiotics, such as aminoglycosides, beta-lactams, fluoroquinolones, and macrolides. The ARGs in representative sources of pollution (e.g., a sewage treatment plant and swine feedlot) were also analyzed for their differences in ARG profiles from relatively pristine Tibet environment. Different from the ARGs in Tibet, the majority of the ARGs in the active sludge and swine feces samples were more specific to a single antibiotic (e.g., aminoglycosides, chloramphenicols, macrolides, tetracyclines, etc.). In particular, the total abundance of ARG in the swine feces samples was at least 30 times higher than those in the animal waste samples from Tibet.

The existence of ARGs in environmental habitats subject to minimal anthropogenic impacts supports the view that antibiotic resistance originated naturally before the modern age of antibiotics. ${ }^{10,12,19,44}$ Nevertheless, whole profiles of antibiotic resistome in the natural environment were seldom delineated, probably due to the enormous variety of ARGs. With rapid development of high throughput sequencing techniques, functional metagenomics was beneficial to the finding of novel ARGs in the environment, ${ }^{45}$ and sequencingbased descriptive metagenomics used in this study was more employed to profile well-recognized ARGs in various samples. ${ }^{30}$ The antibiotic resistance profile of Tibet showed that ARGs in pristine environments were frequently connected with the majority of the antibiotics currently in use or out of use, as observed in the South China Sea. ${ }^{44}$ Furthermore, environmental microflora in the Tibetan environment tend to develop antibiotic resistance in an economic way, with most of the ARGs not restricted to resistance to a single antibiotic. It could not be denied that enzyme-mediated resistance to a single antibiotic (e.g., beta-lactams and macorlides) was found for culturable bacterial strains from isolated caves and the deep oceans. $^{10,11}$ However, resistance mechanisms specific to 
$\mathbf{A}$
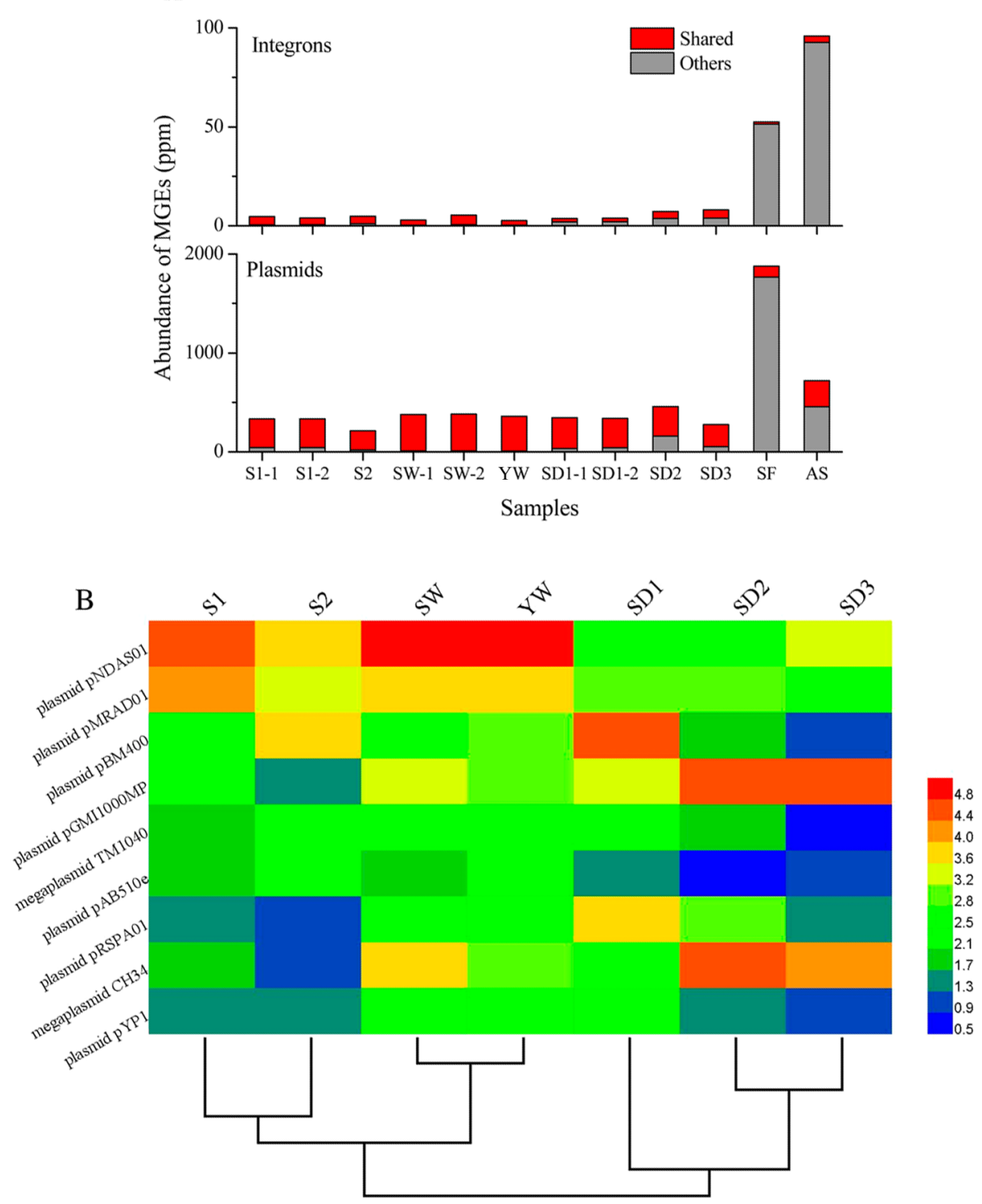

Figure 4. Abundance of MGEs in the soils, animal wastes, and sediments of Tibet and representative sources of pollution (A) and heat map of plasmids in the samples of Tibet (B). Sample identities are the same as those in Figure 1. "Shared" represents the MGEs that were found in all three types of samples, and "Others" refers to the MGEs that were found in only one or two types of samples. The abundance of plasmid-like tags in each of the samples was used for the HCA analysis. The natural logarithm of the number of plasmid-like tags was used to depict the heat map. The accession number of the plasmids in the NCBI was NC_014621.1 (plasmid pYP1), NC_007974.2 (CH34 megaplasmid), NC_009429.1 (plasmid pRSPA01), NC_013859.1 (plasmid pAB510e), NC_008043.1 (TM1040 megaplasmid), NC_003296.1 (plasmid pGMI1000MP), NC_004604.2 (plasmid pBM400), NC_010510.1 (plasmid pMRAD̄01), and NC_014211.1 (plasmid pNDAS01).

multiple antibiotics are dominant and abundant in the pristine environment. ${ }^{44}$ Assuming that energy is limited in unique environment, it is reasonable for microbes to select the most efficient mode of coping with various environmental stresses in terms of both maintenance cost and functional versatility. ${ }^{46,47}$

Figure $2 \mathrm{~B}$ shows beta-lactam resistance gene subtypes in the Tibetan samples and in the representative sources of ARG pollution (e.g., swine feces and active sludge). Six subtypes related to beta-lactam resistance were identified in Tibet, with a preponderance of mex $\mathrm{B}$ and $a c r \mathrm{~B}$. The resistance to beta-lactam might be achieved mainly via an efflux mechanism conferred by acr $\mathrm{B}, \operatorname{mex} \mathrm{B}$, and tol $\mathrm{C}$. In comparison to the Tibet samples, the lactamase-mediated inactivation mechanism was more prevalent than other mechanisms in the typical sources of ARG pollution. Almost $99 \%$ of the beta-lactam resistance in the swine feces was achieved via five subtypes of lactamases. Eight subtypes of lactamases in the active sludge accounted for approximately $54 \%$ of the resistance to beta-lactam.

Exploring baseline profiles of ARGs in the pristine environment could provide the clues to at what primitive state the evolution and development of contemporary antibiotic resistome began. Comparative studies have suggested that ARGs in the human-impacted environments have been diversifying in terms of both gene sequences and resistance mechanisms, as shown in Figure S2 (SI). Therefore, microbes are surviving under much harsher conditions than in the preantibiotic era because a large amount of anthropogenic antibiotics are released into the ambient environment. ${ }^{44,48}$ For example, a significant fraction of lactamase-encoded genes in the ARDB database (a total of 259 subtypes) are the dominant 
resistance mechanism to anthropogenic beta-lactams. ${ }^{28} \mathrm{Sim}$ ilarly, the sulfonamide and tetracycline resistance genes that were prevalent in typical sources of ARG pollution were seldom found in relatively pristine Tibet. ${ }^{44}$ Although ARG profiles have changed substantially since the broad use of anthropogenic antibiotics, the efflux pump as a common way of clearing intracellular toxins is still important because of the essential role it plays in bacterial resistance to antibiotics ${ }^{49,50}$ as well as to pollutants with antimicrobial activity. ${ }^{51-53}$

Comparison of ARG Profiles among Different Environmental Habitats of Tibet. No significant differences in the total number of genotypes, sequence diversities, and resistance mechanisms were observed among the three environmental habitats in Tibet (Figure S2 in the SI). However, about $60 \%$ of the ARG subtypes that were identified in Tibet (a total of 47) were present only in a single environmental habitat (Figure 3A). Thirteen subtypes of ARGs were concurrently detected in the soils, animal wastes, and sediments of Tibet. Here, four subtypes (bacA, mex $\mathrm{B}, \operatorname{mex} \mathrm{F}$, and mexW) were the most popular and were predominant over other subtypes, which were linked to different types of resistance. The protein product of the bacA gene can bypass the inhibition of the isoprenyl pyrophosphate dephosphorylation caused by bacitracin (polypeptide). ${ }^{54}$ Interestingly, in spite of substantial differences in essential properties (e.g., salinity) between Lake Namco of Tibet and the SCS, the bacA gene was prevalent and dominant in the sediments of both pristine aquatic environments. ${ }^{44}$ A great difference in ARG subtype was also observed between the samples of Tibet and typical sources of ARG pollution. More than $80 \%$ of ARG subtypes identified in the SF and AS were not found in the samples of Tibet. It suggests that antibiotic resistome has been substantially changed since anthropogenic antibiotics are widely used.

Furthermore, the relationships between the samples were further explored using a PCA approach according to the relative abundance of resistance types (Figure 3B). The first two principal components accounted for approximately $66.8 \%$ of the total variance in antibiotic resistance types among all samples. The samples collected from representative sources of ARG pollution were significantly separated from those from the relatively pristine Tibetan environment. The samples from the same environmental habitats were usually grouped together due to the similarity of their antibiotic resistance profiles.

The ARGs of different environmental habitats in Tibet evolved in the absence of substantial anthropogenic impacts. Natural gene flows, in the form of both bacterial hosts and/or gene material, undoubtedly exist between different environmental habitats. They probably contributed to the copresence of some ARG subtypes in all of the samples from different habitats. However, bacterial communities residing in different environmental habitats in Tibet also maintained their respective traits of antibiotic resistance profiles. Different from the Tibetan environment, natural gene flows between different habitats in the human-impacted environments were always considerably disturbed by intensive human activities. Composting and fertilizing operations accelerated the flows of ARGs from animal intestines to the soils. ${ }^{29,55}$ Huge amounts of ARGs and ARB were released from urban settings to nearby aquatic environments via the discharges of sewage treatment plants. ${ }^{28,56}$ As a consequence of anthropogenic impacts, accelerating gene flows could diminish differences among the antibiotic resistance profiles of various environmental habitats; thus, it is almost impossible to retrieve original resistance profile of each of habitats in a highly human-impacted environment.

Occurrence and Abundance of MGEs in Tibet. Sequencing results were aligned against the databases of integrons and plasmids to investigate their occurrence and abundance in Tibet (Figure 4A). A large variety of integrons and plasmids were found in Tibet, but there were no great variations in total abundance among different sample types. The total quantity of plasmid-like tags was substantially greater than that of integron-like ones. A large number of integron-like tags were annotated as various recombinase-encoded genes (e.g., intI, tni, etc.). The relative abundance of recombinaserelated tags over the total number of integron-like tags was considerably higher in the soils and sediments (e.g., $22.5 \%$ and $33.8 \%$ ) than in the animal wastes (e.g., 6.1\%). The plasmids that were concurrently found in all environmental habitats accounted for about $75 \%$ of the total plasmid-like tags in the Tibetan samples. Approximately $70 \%$ of integrons were shared by all samples of Tibet. The abundance of both plasmids and integrons in representative pollution sources (SW and AS) was significantly higher than those in the samples collected from Tibet $(p<0.01)$. Moreover, the plasmids and integrons coexisting in all samples of Tibet only took up less than $36 \%$ of those in the SW and AS.

A heat map of the abundance of the top nine major plasmids demonstrated that distinct patterns were observed among different environmental habitats in Tibet (Figure 4B). Samples were classified according to the trait of the plasmid genome, which was in good agreement with different environmental habitats. Representative profiles of the plasmid genome in the soils, animal wastes, and sediments of Tibet are shown in Figure S3 of the SI. The pNDAS01 and pMRAD01 plasmids were predominant in the soils, whereas the pGMI1000MP plasmid and $\mathrm{CH} 34$ megaplasmid were preponderant in the sediments. The fact that whole profiles of MGEs were present in the pristine environments has rarely been explored, as well as the exact factors that led to the formation of typical profiles of MGEs in different environmental habitats. ${ }^{57}$ However, bacterial communities often benefit from MGE-carrying genes functioning in harsh conditions. ${ }^{58,59}$ Consequently, the distribution patterns of these MGE-carrying genes among bacteria hosts became an important influencing factor to the composition and structure of bacterial community, vice versa.

Horizontal Transferability of ARGs in Tibet. In order to evaluate the potential for ARGs to be transferred among bacteria in Tibet, ARG-like tags were extracted from all sequencing data sets and aligned against the databases of integrons and plasmids. The results demonstrated that a tiny fraction of ARG-like tags in Tibet were also assigned to the integrons and plasmids. Three tags of ARGs (e.g., aadA, sulI, and tet $\mathrm{G}$ ) were identified in the gene cassettes of the integrons, and approximately $2.0 \%$ of all ARG-like tags in all samples were carried by the plasmids (Figure 5). In case of plasmid-carrying ARGs being missed because of inadequate sequencing depth, the full-length sequences of the plasmids that had been concurrently identified in all of the Tibet samples were extracted from the plasmid database, and then were aligned against the ARDB database. These plasmids only carried complete sequences of mex $\mathrm{B}$ and mex $\mathrm{C}$. By contrast, relative percentages of MEGs-related ARGs in the SF and AS were significantly higher than those in the samples from Tibet $(p<$ $0.01)$. For instance, approximately half of the ARGs in the AS were potentially associated with the plasmids. 


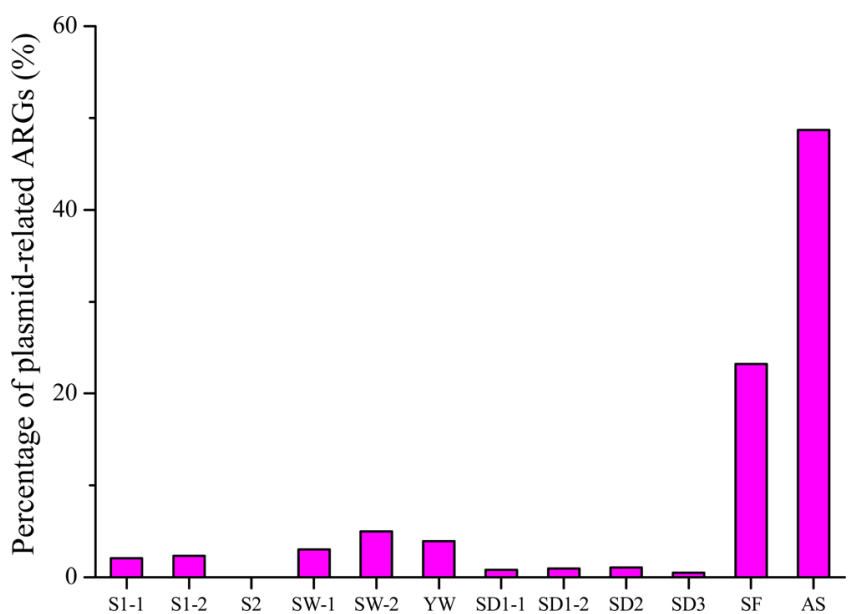

Figure 5. Relative percentages of ARGs potential related to plasmids over the total ARGs in the Tibetan samples and representative sources of ARG pollution.

ARGs are disseminated in the human-impacted environments mainly via promiscuous gene transfers between genetic locations or between bacterial hosts under elevated antibiotic stresses, viz., horizontal gene transfers. ${ }^{22,23}$ Such transfers of ARGs required MGEs as the carriers. As a result, significant relationships between ARGs and MGEs in terms of diversity and abundance were frequently observed in different environmental niches. ${ }^{44,60,61}$ Integrons consist of a site-specific recombinase gene and a shuffled gene cassette. Class I integrons are ubiquitous in environmental microflora, ${ }^{62-65}$ as was detectable in all samples from Tibet. It has been highlighted that gene cassettes in human-impacted settings are recombinant with diverse and abundant ARGs responding to anthropogenic antibiotics. ${ }^{66,67}$ yet this is not the case in pristine environments. The present study showed that ARGlike genes were sparse in the integron genomes. As a MGE with larger size, the plasmids identified in the Tibetan samples were also quite devoid of ARGs. An increasing number of studies have demonstrated that ARB in the niches unaffected by human activities probably possess a wide array of clinically associated and novel ARGs. ${ }^{68}$ Nevertheless, transformation assays often showed that antibiotic resistance of these ARB was not transferable to susceptible Escherichia coli. ${ }^{69}$ Our results revealed that the MGEs common found in the Tibetan environment lack ARGs, indicating that most of the ARGs in Tibet could have a low potential to transfer between bacterial hosts. A substantial number of potentially MGEs-carrying ARGs in the swine feces and active sludge could be the consequence of the horizontal transfer of genes from the heavy use of anthropogenic antibiotics, and would facilitate the continual transfer of ARGs between bacterial hosts.

Environmental Implications. Diverse ARGs originating from natural environments were found in the remote Tibetan environment. The whole profiles of ARGs in Tibet differed significantly from those of representative ARG pollution sources in terms of both major subtypes and resistance mechanisms. It suggests that the modern resistome has evolved significantly from the preantibiotic age or minimally humanimpacted environment since bacterial communities in the human-impacted environment endure a wide occurrence of anthropogenic antibiotics. ARGs found in the Tibetan environment are seldom harbored in the MEGs, which implies that ARGs can hardly be transferred horizontally between bacterial hosts in the absence of antibiotic stress in relatively pristine environments. In the human-impacted settings, MGEs enriched with diverse ARGs can greatly facilitate the acquisition of antibiotic resistance by bacteria.

\section{ASSOCIATED CONTENT}

\section{S Supporting Information}

The Supporting Information is available free of charge on the ACS Publications website at DOI: 10.1021/acs.est.6b00619.

The accession number of sequencing data used in this study, relative percentages of ARG resistance types, subtypes and sequence diversity of ARGs and resistance mechanism, and representative profiles of plasmid genome in the soils, animal wastes and sediments of Tibet and in typical pollution sources (PDF)

\section{AUTHOR INFORMATION}

\section{Corresponding Author}

*Phone: +852-27666041; fax:+852-23346389; e-mail: cexdli@ polyu.edu.hk.

\section{Author Contributions}

B.W.C. and X.D.L. designed this study; B.W.C. and Y.W.W. collected the samples in the field; B.W.C. and K.Y. measured antibiotic concentrations; B.W.C., Y.Y., X.C., and K.Y. analyzed the samples and data; B.W.C. and X.D.L. drafted the paper; and all of the authors contributed to revising the manuscript and approving the final version. We declare that they have no competing financial interests.

\section{Notes}

The authors declare no competing financial interest.

\section{ACKNOWLEDGMENTS}

This work was supported by the Provincial Natural Science Foundation of Guangdong, China (2014A030313195), the Area of Excellence (AoE) project under Grant No. AoE/P-04/ 2004 from the University Grants Council of Hong Kong, the State Key Laboratory of Environmental Chemistry and Ecotoxicology, Research Center for Eco-Environmental Sciences, Chinese Academy of Sciences (KF2014-01), and the National Natural Science Foundation of China (21177162). We are very grateful for the help from Prof. Shichang Kang, and Drs.. Zhiyuan Cong, Chengding $\mathrm{Li}$ and Pengfei Chen of Institute of Tibetan Plateau Research, Chinese Academy of Sciences on the field sampling of sediment samples from Lake Namco, Tibet.

\section{REFERENCES}

(1) Rachakonda, S.; Cartee, L. Challenges in antimicrobial drug discovery and the potential of nucleoside antibiotics. Curr. Med. Chem. 2004, 11 (6), 775-793.

(2) Sommer, M. O.; Dantas, G.; Church, G. M. Functional characterization of the antibiotic resistance reservoir in the human microflora. Science 2009, 325 (5944), 1128-1131.

(3) Livermore, D. M. Has the era of untreatable infections arrived? J. Antimicrob. Chemother. 2009, 64 (4), 29-36.

(4) D’Costa, V. M.; McGrann, K. M.; Hughes, D. W.; Wright, G. D. Sampling the antibiotic resistome. Science 2006, 311 (5759), 374-377.

(5) Nathan, C. Antibiotics at the crossroads. Nature 2004, 431 (7011), 899-902.

(6) Wenzel, R. P.; Edmond, M. B. Managing antibiotic resistance. N. Engl. J. Med. 2000, 343 (26), 1961-1963.

(7) Hughes, V. M.; Datta, N. Conjugative Plasmids in Bacteria of the Pre-Antibiotic Era. Nature 1983, 302 (5910), 725-726. 
(8) Davis, C. E.; Anandan, J. Evolution of R Factor. A Study of a Preantibiotic Community in Borneo. N. Engl. J. Med. 1970, 282 (3), $117-122$.

(9) Knapp, C. W.; Dolfing, J.; Ehlert, P. A. I.; Graham, D. W. Evidence of Increasing Antibiotic Resistance Gene Abundances in Archived Soils since 1940. Environ. Sci. Technol. 2010, 44 (2), 580587.

(10) Bhullar, K.; Waglechner, N.; Pawlowski, A.; Koteva, K.; Banks, E. D.; Johnston, M. D.; Barton, H. A.; Wright, G. D. Antibiotic Resistance Is Prevalent in an Isolated Cave Microbiome. PLoS One 2012, 7 (4), e34953.

(11) Toth, M.; Smith, C.; Frase, H.; Mobashery, S.; Vakulenko, S. An Antibiotic-Resistance Enzyme from a Deep-Sea Bacterium. J. Am. Chem. Soc. 2010, 132 (2), 816-823.

(12) Brown, M. G.; Balkwill, D. L. Antibiotic Resistance in Bacteria Isolated from the Deep Terrestrial Subsurface. Microb. Ecol. 2009, 57 (3), 484-493.

(13) Newman, D. J.; Cragg, G. M.; Snader, K. M. Natural products as sources of new drugs over the period 1981-2002. J. Nat. Prod. 2003, 66 (7), 1022-1037.

(14) Baltz, R. H. Renaissance in antibacterial discovery from actinomycetes. Curr. Opin. Pharmacol. 2008, 8 (5), 557-563.

(15) Jang, K. H.; Nam, S. J.; Locke, J. B.; Kauffman, C. A.; Beatty, D. S.; Paul, L. A.; Fenical, W. Anthracimycin, a Potent Anthrax Antibiotic from a Marine-Derived Actinomycete. Angew. Chem., Int. Ed. 2013, 52 (30), 7822-7824

(16) Dantas, G.; Sommer, M. O. A.; Oluwasegun, R. D.; Church, G. M. Bacteria Subsisting on Antibiotics. Science 2008, 320 (5872), 100103.

(17) Cordero, O. X.; Wildschutte, H.; Kirkup, B.; Proehl, S.; Ngo, L.; Hussain, F.; Le Roux, F.; Mincer, T.; Polz, M. F. Ecological populations of bacteria act as socially cohesive units of antibiotic production and resistance. Science 2012, 337 (6099), 1228-1231.

(18) Wright, G. D. The antibiotic resistome: the nexus of chemical and genetic diversity. Nat. Rev. Microbiol. 2007, 5 (3), 175-186.

(19) D’Costa, V. M.; King, C. E.; Kalan, L.; Morar, M.; Sung, W. W. L.; Schwarz, C.; Froese, D.; Zazula, G.; Calmels, F.; Debruyne, R.; Golding, G. B.; Poinar, H. N.; Wright, G. D. Antibiotic resistance is ancient. Nature 2011, 477 (7365), 457-461.

(20) Xiong, W.; Sun, Y.; Zhang, T.; Ding, X.; Li, Y.; Wang, M.; Zeng, Z. Antibiotics, Antibiotic Resistance Genes, and Bacterial Community Composition in Fresh Water Aquaculture Environment in China. Microb. Ecol. 2015, 70 (2), 425-432.

(21) Chen, B. W.; Liang, X. M.; Huang, X. P.; Zhang, T.; Li, X. D. Differentiating anthropogenic impacts on ARGs in the Pearl River Estuary by using suitable gene indicators. Water Res. 2013, 47 (8), 2811-2820.

(22) Gootz, T. D. The global problem of antibiotic resistance. Crit. Rev. Immunol. 2010, 30 (1), 79-93.

(23) Bellanger, X.; Guilloteau, H.; Bonot, S.; Merlin, C. Demonstrating plasmid-based horizontal gene transfer in complex environmental matrices: a practical approach for a critical review. Sci. Total Environ. 2014, 493, 872-82.

(24) Elsaied, H.; Stokes, H. W.; Kitamura, K.; Kurusu, Y.; Kamagata, Y.; Maruyama, A. Marine integrons containing novel integrase genes, attachment sites, attI, and associated gene cassettes in polluted sediments from Suez and Tokyo Bays. ISME J. 2011, 5 (7), 11621177.

(25) Liu, X. B.; Yao, T. D.; Kang, S. C.; Jiao, N. A. Z.; Zeng, Y. H.; Liu, Y. Q. Bacterial Community of the Largest Oligosaline Lake, Namco on the Tibetan Plateau. Geomicrobiol. J. 2010, 27 (8), 669682.

(26) Wang, J. B.; Zhu, L. P.; Daut, G.; Ju, J. T.; Lin, X.; Wang, Y.; Zhen, X. L. Investigation of bathymetry and water quality of Lake Nam Co, the largest lake on the central Tibetan Plateau, China. Limnology 2009, 10 (2), 149-158.

(27) Xia, Z. H.; Xu, B. Q.; Mugler, I.; Wu, G. J.; Gleixner, G.; Sachse, D.; Zhu, L. P. Hydrogen isotope ratios of terrigenous $n$-alkanes in lacustrine surface sediment of the Tibetan Plateau record the precipitation signal. Geochem. J. 2008, 42 (4), 331-338.

(28) Yang, Y.; Li, B.; Ju, F.; Zhang, T. Exploring Variation of Antibiotic Resistance Genes in Activated Sludge over a Four-Year Period through a Metagenomic Approach. Environ. Sci. Technol. 2013, 47 (18), 10197-10205.

(29) Zhu, Y. G.; Johnson, T. A.; Su, J. Q.; Qiao, M.; Guo, G. X.; Stedtfeld, R. D.; Hashsham, S. A.; Tiedje, J. M. Diverse and abundant antibiotic resistance genes in Chinese swine farms. Proc. Natl. Acad. Sci. U. S. A. 2013, 110 (9), 3435-3440.

(30) Li, B.; Yang, Y.; Ma, L.; Ju, F.; Guo, F.; Tiedje, J. M.; Zhang, T. Metagenomic and network analysis reveal wide distribution and cooccurrence of environmental antibiotic resistance genes. ISME J. 2015, 9 (11), 2490-2502.

(31) Liang, X. M.; Chen, B. W.; Nie, X. P.; Shi, Z.; Huang, X. P.; Li, $\mathrm{X}$. D. The distribution and partitioning of common antibiotics in water and sediment of the Pearl River Estuary, South China. Chemosphere 2013, 92 (11), 1410-1416.

(32) Altschul, S. F.; Madden, T. L.; Schaffer, A. A.; Zhang, J.; Zhang, Z.; Miller, W.; Lipman, D. J. Gapped BLAST and PSI-BLAST: a new generation of protein database search programs. Nucleic Acids Res. 1997, 25 (17), 3389-402.

(33) Mackelprang, R.; Waldrop, M. P.; DeAngelis, K. M.; David, M. M.; Chavarria, K. L.; Blazewicz, S. J.; Rubin, E. M.; Jansson, J. K. Metagenomic analysis of a permafrost microbial community reveals a rapid response to thaw. Nature 2011, 480 (7377), 368-371.

(34) Huson, D. H.; Auch, A. F.; Qi, J.; Schuster, S. C. MEGAN analysis of metagenomic data. Genome Res. 2007, 17 (3), 377-386.

(35) Liu, B.; Pop, M. ARDB-Antibiotic Resistance Genes Database. Nucleic Acids Res. 2009, 37, D443-D447.

(36) Moura, A.; Soares, M.; Pereira, C.; Leitao, N.; Henriques, I.; Correia, A. INTEGRALL: a database and search engine for integrons, integrases and gene cassettes. Bioinformatics 2009, 25 (8), 1096-1098.

(37) Zhang, T.; Zhang, X. X.; Ye, L. Plasmid Metagenome Reveals High Levels of Antibiotic Resistance Genes and Mobile Genetic Elements in Activated Sludge. PLoS One 2011, 6 (10), e26041.

(38) Zhang, G.; Niu, F.; Ma, X.; Liu, W.; Dong, M.; Feng, H.; An, L.; Cheng, G. Phylogenetic diversity of bacteria isolates from the QinghaiTibet Plateau permafrost region. Can. J. Microbiol. 2007, 53 (8), $1000-1010$

(39) Yun, J. L.; Ju, Y. W.; Deng, Y. C.; Zhang, H. X. Bacterial Community Structure in Two Permafrost Wetlands on the Tibetan Plateau and Sanjiang Plain, China. Microb. Ecol. 2014, 68 (2), 360369.

(40) Rui, J.; Li, J.; Wang, S.; An, J.; Liu, W. T.; Lin, Q.; Yang, Y.; He, Z.; Li, X. Responses of Bacterial Communities to Simulated Climate Changes in Alpine Meadow Soil of the Qinghai-Tibet Plateau. Appl. Environ. Microbiol. 2015, 81 (17), 6070-6077.

(41) Jiang, H.; Dong, H.; Yu, B.; Liu, X.; Li, Y.; Ji, S.; Zhang, C. L. Microbial response to salinity change in Lake Chaka, a hypersaline lake on Tibetan plateau. Environ. Microbiol. 2007, 9 (10), 2603-2621.

(42) Liu, Y. Q.; Yao, T. D.; Jiao, N. Z.; Zhu, L. P.; Hu, A. Y.; Liu, X. B.; Gao, J.; Chen, Z. Q. Salinity Impact on Bacterial Community Composition in Five High-Altitude Lakes from the Tibetan Plateau, Western China. Geomicrobiol. J. 2013, 30 (5), 462-469.

(43) Tan, H. M.; Deng, Q. L.; Cao, L. X. Ruminant feces harbor diverse uncultured symbiotic actinobacteria. World J. Microbiol. Biotechnol. 2014, 30 (3), 1093-1100.

(44) Chen, B. W.; Yang, Y.; Liang, X. M.; Yu, K.; Zhang, T.; Li, X. D. Metagenomic Profiles of Antibiotic Resistance Genes (ARGs) between Human Impacted Estuary and Deep Ocean Sediments. Environ. Sci. Technol. 2013, 47 (22), 12753-12760.

(45) Munck, C.; Albertsen, M.; Telke, A.; Ellabaan, M.; Nielsen, P. H.; Sommer, M. O. Limited dissemination of the wastewater treatment plant core resistome. Nat. Commun. 2015, 6, 8452.

(46) Wright, G. D. The antibiotic resistome: the nexus of chemical and genetic diversity. Nat. Rev. Microbiol. 2007, 5 (3), 175-186.

(47) Fredrickson, J. K.; Garland, T. R.; Hicks, R. J.; Thomas, J. M.; Li, S. W.; Mcfadden, K. M. Lithotrophic and heterotrophic bacteria in 
deep subsurface sediments and their relation to sediment properties. Geomicrobiol. J. 1989, 7 (1-2), 53-66.

(48) Zhang, X. X.; Zhang, T.; Fang, H. H. Antibiotic resistance genes in water environment. Appl. Microbiol. Biotechnol. 2009, 82 (3), 397414.

(49) Li, X. Z.; Plesiat, P.; Nikaido, H. The Challenge of EffluxMediated Antibiotic Resistance in Gram-Negative Bacteria. Clin. Microbiol. Rev. 2015, 28 (2), 337-418.

(50) Bellido, J. L. M.; Guirao, G. Y.; Zufiaurre, N. G.; Manzanares, A. A. Efflux-mediated antibiotic resistance in Gram-positive bacteria. Rev. Med. Microbiol. 2002, 13 (1), 1-13.

(51) Silver, S. Bacterial resistances to toxic metal ions-a review. Gene 1996, 179 (1), 9-19.

(52) Ryan, B. M.; Dougherty, T. J.; Beaulieu, D.; Chuang, J.; Dougherty, B. A.; Barrett, J. F. Efflux in bacteria: what do we really know about it? Expert Opin. Invest. Drugs 2001, 10 (8), 1409-1422.

(53) Taylor, D. L.; Bina, X. W. R.; Bina, J. E. Vibrio cholerae vexH Encodes a Multiple Drug Efflux Pump That Contributes to the Production of Cholera Toxin and the Toxin Co-Regulated Pilus. PLoS One 2012, 7 (5), e38208.

(54) El Ghachi, M.; Bouhss, A.; Blanot, D.; Mengin-Lecreulx, D. The bacA gene of Escherichia coli encodes an undecaprenyl pyrophosphate phosphatase activity. J. Biol. Chem. 2004, 279 (29), 30106-30113.

(55) Su, J. Q.; Wei, B.; Ou-Yang, W. Y.; Huang, F. Y.; Zhao, Y.; Xu, H. J.; Zhu, Y. G. Antibiotic Resistome and Its Association with Bacterial Communities during Sewage Sludge Composting. Environ. Sci. Technol. 2015, 49 (12), 7356-7363.

(56) Wu, D.; Huang, Z. T.; Yang, K.; Graham, D.; Xie, B. Relationships between Antibiotics and Antibiotic Resistance Gene Levels in Municipal Solid Waste Leachates in Shanghai, China. Environ. Sci. Technol. 2015, 49 (7), 4122-4128.

(57) Martinez-Rosales, C.; Fullana, N.; Musto, H.; Castro-Sowinski, S. Antarctic DNA moving forward: genomic plasticity and biotechnological potential. FEMS Microbiol. Lett. 2012, 331 (1), 1-9.

(58) Beuls, E.; Modrie, P.; Deserranno, C.; Mahillon, J. High-Salt Stress Conditions Increase the pAW63 Transfer Frequency in Bacillus thuringiensis. Appl. Environ. Microbiol. 2012, 78 (19), 7128-7131.

(59) Yang, X. W.; Jian, H. H.; Wang, F. P. pSW2, a Novel LowTemperature-Inducible Gene Expression Vector Based on a Filamentous Phage of the Deep-Sea Bacterium Shewanella piezotolerans WP3. Appl. Environ. Microbiol. 2015, 81 (16), 5519-5526.

(60) Luo, Y.; Mao, D. Q.; Rysz, M.; Zhou, D. X.; Zhang, H. J.; Xu, L.; Alvarez, P. J. J. Trends in Antibiotic Resistance Genes Occurrence in the Haihe River, China. Environ. Sci. Technol. 2010, 44 (19), 72207225.

(61) Chen, B. W.; Liang, X. M.; Nie, X. P.; Huang, X.; Zou, S. C.; Li, $\mathrm{X}$. D. The role of class I integrons in the dissemination of sulfonamide resistance genes in the Pearl River and Pearl River Estuary, South China. J. Hazard. Mater. 2015, 282, 61-67.

(62) Di Conza, J. A.; Gutkind, G. O. [Integrons: gene collectors]. Rev. Argent Microbiol. 2010, 42 (1), 63-78.

(63) Shah, S. Q. A.; Colquhoun, D. J.; Nikuli, H. L.; Sorum, H. Prevalence of Antibiotic Resistance Genes in the Bacterial Flora of Integrated Fish Farming Environments of Pakistan and Tanzania. Environ. Sci. Technol. 2012, 46 (16), 8672-8679.

(64) Yan, H.; Li, L.; Zong, M. H.; Alam, M. J.; Shinoda, S.; Shi, L. Occurrence and Characteristics of Class 1 and 2 Integrons in Clinical Bacterial Isolates from Patients in South China. J. Health Sci. 2010, 56 (4), 442-450.

(65) Ndi, O. L.; Barton, M. D. Incidence of class 1 integron and other antibiotic resistance determinants in Aeromonas spp. from rainbow trout farms in Australia. J. Fish Dis. 2011, 34 (8), 589-599.

(66) Wright, M. S.; Baker-Austin, C.; Lindell, A. H.; Stepanauskas, R.; Stokes, H. W.; McArthur, J. V. Influence of industrial contamination on mobile genetic elements: class 1 integron abundance and gene cassette structure in aquatic bacterial communities. ISME J. 2008, 2 (4), 417-428
(67) Partridge, S. R.; Tsafnat, G.; Coiera, E.; Iredell, J. R. Gene cassettes and cassette arrays in mobile resistance integrons. FEMS Microbiol. Rev. 2009, 33 (4), 757-784.

(68) Cytryn, E. The soil resistome: The anthropogenic, the native, and the unknown. Soil Biol. Biochem. 2013, 63, 18-23.

(69) Fredrickson, J. K.; Hicks, R. J.; Li, S. W.; Brockman, F. J. Plasmid incidence in bacteria from deep subsurface sediments. Appl. Environ. Microbiol. 1988, 54 (12), 2916-2923. 